\title{
Comparative Study between Cubic and Non-Cubic Equations of State Using Carnahan-Starling Repulsive Term: Application of Temperature-Dependent Alpha and Beta Functions
}

\author{
R. F. Checoni ${ }^{1}$ and M. Aznar ${ }^{2}$ \\ ${ }^{1}$ Chemical Engineering Program - Texas A\&M University at Qatar - Doha - Qatar \\ ${ }^{2}$ Faculdade de Engenharia Química, Universidade Estadual de Campinas - UNICAMP - Brazil. \\ E-mail: ${ }^{1}$ rfcheconi@yahoo.com.br
}

\begin{abstract}
Studies on phase equilibria data behavior of pure substances are motivation to the researchers due to importance of these data for the scientific and industrial applications. Several EOS were proposed and its modifications have been made, whose aim is to improve the correlation between experimental and calculated thermophysical properties. This work proposes a comparative study between the PVT calculated data using cubic and non-cubic equations of state, in which its original repulsive term is substituted by the Carnahan-Starling hard-sphere repulsive term; furthermore, generalized expression to calculate $\alpha(T r, \omega)$ and $\beta(T r, \omega)$ functions are used. Experimental data of vapor pressure for various pure compounds were compared to the calculated vapor pressure data showing satisfactory agreement, when this proposed modification is employed.
\end{abstract}

Keywords: Equation of state; generalized alpha and beta functions; modeling; pure compounds.

\section{Introduction}

Equations of state (EOSs) were developed to predict the phase equilibria of the substances (pure or mixtures), using its experimental data of pressure, temperature, and volume. However, due to particular characteristic of each compounds group, an equation of state is unable to describe the phase equilibria for all substances existing. In general, several researcher group chosen the van der Waals EOS type, in which are represented conceptually by sum of repulsive and attractive terms, showed by

$$
p=\frac{R T}{(V-b)}-\frac{a}{V^{2}+x b V+y b^{2}}
$$

wherein $p, V, T, R, a$ and $b$ are pressure, molar volume, temperature, universal constant of gases, attraction parameter, and molecular volume parameter, respectively. The parameters $x$ and $y$ in Eq. (1) are characteristic of each EOS: if $x=y=0$, this equation is the van der Waals EOS (Abbot [1]); if $x=1$ and $y=0$, this equation is that proposed by Soave [2] and if $x=2$ and $y=-1$, the equation is that one proposed by Peng and Robinson [3]. This last one equation of state is considered the most adequate and employed in to the prediction of the phase equilibria behavior as well as in the design and optimization of industrial equipments and processes [4].

In spite of its adequate format, several modifications in the original format of Peng and Robinson EOS [3] have been proposed such as: changes in its original alpha function, used to calculate attraction parameter temperature dependent [5-6], as well as to consider the co-volume temperature dependent [7-9]. All of resultant EOSs contribute for improvement of the phase equilibria calculations for both pure compounds and mixtures. A variety of studies involving cubic EOSs suggest that it is possible to generalize the adjustable parameters of various pure compounds [10-11]. It is common for this generalization to account for mathematical expressions (alpha-functions), whose relation is established with the temperature and acentric factor of the pure compounds; some examples of alpha functions can be found in [12-14].

In general, the modification of a cubic EOS consists in to change the attractive term, as mentioned above; but it is possible to modify also the repulsive term, as showed in [4] to improve the representation of the thermophysical properties of compounds. Besides to these works the repulsive term proposed in [15] widely figures in the literature as accurate and physically consistent term to represent the molecular repulsions contributions in the EOSs [16-18].

Taking into consideration the repulsive term proposed in [15], besides to temperature dependency of the attraction and co-volume parameters, by using a modified version of the generalized alpha function [12], and accounting the values 2 and -2 for the $x$ and $y$, respectively, in the second term of the right side of Eq. (1), the experimental data of vapor pressure of industrially important non-polar pure compounds are correlated, whose values were obtained from [19-22]. The results obtained are compared with those presented by the cubic equation of state proposed in [5] and using slightly derivations. Moreover, two groups of adjustable parameters are presented: one specific to each pure compound and other generalized to all compounds studied here. The advantages in to use one or other parameters group are discussed.

\section{Equation of State}

Starting of the Eq. (1), the attractive and repulsive parameters ( $a$ and $b$ ) are accounted temperature dependents; the values of $x$ and $y$ adopted are 2 and -2 , respectively. Furthermore, repulsive term of this equation is replaced by 
the hard-sphere repulsion term developed by [15]. These considerations result in the following expression:

$$
p=\frac{R T\left(1+y+y^{2}-y^{3}\right)}{V(1-y)^{3}}-\frac{a(T)}{V^{2}+2 V b(T)-2[b(T)]^{2}}
$$

in which $y=b(T) /(4 V)$. Considering that, at the critical conditions, the first and second derivatives of pressure in relation to volume are zero [3], it is possible to obtain

$$
\begin{aligned}
& a_{c}=0.5300 \frac{R^{2} T c^{2}}{P c} \\
& b_{c}=0.1172 \frac{R T c}{P c}
\end{aligned}
$$

At temperatures other than the critical conditions, yet

$$
\begin{aligned}
& a(T)=a_{c} \cdot \alpha(\operatorname{Tr}, \omega) \\
& b(T)=b_{c} \cdot \beta(T r, \omega)
\end{aligned}
$$

wherein $a(T)$ and $b(T)$ are the attraction and co-volume parameters, respectively, both temperature dependents. With these values, it is possible to calculate $\alpha(\operatorname{Tr}, \omega)$ and $\beta(\operatorname{Tr}, \omega)$ which are functions dimensionless of reduced temperature. It is common in the literature the term $\alpha(\operatorname{Tr}, \omega)$ represented in Eq. (5) to be designed by alpha function. So, in this work, $\beta(T r, \omega)$ in Eq. (6) is designated by beta function.

The expressions used to represent the alpha and beta functions $(\alpha(\operatorname{Tr}, \omega)$ and $\beta(\operatorname{Tr}, \omega))$ are based on the modified version of the Mathias and Copeman [12] generalized expression,

$\alpha(\operatorname{Tr}, \omega)=$
$\left(1+A_{1}\left(1-\operatorname{Tr}^{0.5}\right)^{0.5}+A_{2}\left(1-\operatorname{Tr}^{0.5}\right)+A_{3}\left(1-\operatorname{Tr}^{0.5}\right)^{1.5}\right)^{2}$

and

$$
\begin{aligned}
& \beta(T r, \omega)= \\
& \left(1+B_{1}\left(1-\operatorname{Tr}^{0.5}\right)^{0.5}+B_{2}\left(1-\operatorname{Tr}^{0.5}\right)+B_{3}\left(1-\operatorname{Tr}^{0.5}\right)^{1.5}\right)^{2}
\end{aligned}
$$

In these expressions, it is possible to note the maximum value allowed for $\operatorname{Tr}$ is 1.0, since square roots of the negative numbers are indeterminate. So, the proposed EOS is not valid up to the critical conditions. Each function has its proper adjustable parameter group ( $A$ 's and $B$ ' $s$ ) which is structured as a power series in the acentric factor $(\omega)$ truncated at $2^{\text {nd }}$ order

$$
\begin{aligned}
& A_{1}(\omega)=A_{11}+A_{12} \omega+A_{13} \omega^{2} \\
& A_{2}(\omega)=A_{21}+A_{22} \omega+A_{23} \omega^{2} \\
& A_{3}(\omega)=A_{31}+A_{32} \omega+A_{33} \omega^{2} \\
& B_{1}(\omega)=B_{11}+B_{12} \omega+B_{13} \omega^{2}
\end{aligned}
$$

$$
\begin{aligned}
& B_{2}(\omega)=B_{21}+B_{22} \omega+B_{23} \omega^{2} \\
& B_{3}(\omega)=B_{31}+B_{32} \omega+B_{33} \omega^{2}
\end{aligned}
$$

From $\alpha(T r)$ and $\beta(T r)$ values at each $T r$, using Eqs. (5) and (6), it is possible to calculate the $A$ and $B$ adjustable parameters of Eqs. (7) and (8), which are specific of each pure compound; in this way, if various pure compounds are used, various $A$ 's and $B$ 's adjustable parameters groups are obtained. Each pure compound has its particular acentric factor $(\omega)$. So, employing these parameters in Eqs. (9) and (10), generalized alpha and beta functions are obtained in relation to the pure compounds studied in this work.

There are some limiting conditions and general trends which must be satisfied by any equation of state [23]. The first criterion analyzed is to verify if the $\lim P V=R T$, as $P \rightarrow 0$, at any temperature. Considering Eq. (2) multiplied by volume $(V)$

$$
\begin{aligned}
& P V= \\
& \frac{R T\left(1+b / 4 V+(b / 4 V)^{2}-(b / 4 V)^{3}\right)}{(1-b / 4 V)^{3}}-\frac{a}{V+2 b-2 b^{2} / V}
\end{aligned}
$$

the following limit condition is observed at any temperature

$$
\lim _{\substack{V \rightarrow \infty \\ P \rightarrow 0}} P V=R T
$$

since $b /(4 V) \rightarrow 0$ and $a /\left(V+2 b-2\left(b^{2}\right) / V\right) \rightarrow 0$ as $V \rightarrow \infty$.

The second criterion analyzed is to verify if $\lim _{T \rightarrow \infty} V / T=R / P$ at any pressure. Rearranging Eq. (3)

$$
\frac{V}{T}=\frac{R\left(1+b / 4 V+(b / 4 V)^{2}-(b / 4 V)^{3}\right)}{P V(1-b / 4 V)^{3}}-\frac{a V}{P T}
$$

the limit condition below is observed at any pressure

$$
\lim _{\substack{V \rightarrow \infty \\ T \rightarrow \infty}} V / T=R / P
$$

since $b /(4 V) \rightarrow 0$ as $\mathrm{V} \rightarrow \infty$ and $(a V) /(P T) \rightarrow 0$ as $T \rightarrow \infty$.

The third criterion analyzed is associated to the inflexion point of the critical isotherm curve. Mathematically,

$$
\left(\frac{\partial P}{\partial V}\right)_{T_{C}}=0
$$

and

$$
\left(\frac{\partial^{2} P}{\partial V^{2}}\right)_{T_{C}}=0
$$

Applying the proposed Eq. (2) at the critical conditions 


$$
p_{C}=\frac{R T_{C}\left(1+b / 4 V_{C}+\left(b / 4 V_{C}\right)^{2}-\left(b / 4 V_{C}\right)^{3}\right)}{V_{C}\left(1-b / 4 V_{C}\right)^{3}}
$$

and considering the Eqs. (8) and (9) it is possible to obtain expressions for $a, b$ and $R$ in terms of $V_{C}, P_{C}$ and $T_{C}$ :

$$
\begin{aligned}
V_{C} & =2.397 b \\
P_{C} & =\frac{a}{38.59 b^{2}} \\
T_{C} & =\frac{5 a}{22.61 R b}
\end{aligned}
$$

From these expressions it follows that

$$
\begin{aligned}
& b=0.4172 V_{C} \\
& a=6.7168 P_{C} V_{C}^{2} \\
& R=3.5603 \frac{P_{C} V_{C}}{T_{C}} \\
& \text { and } \\
& Z_{C}=\frac{P_{C} V_{C}}{R T_{C}}=0.2809
\end{aligned}
$$

The fourth criterion is to verify if $(P \times T)$ curves (isometrics) are linear, since this behavior is normally verified except at very high densities. Mathematically,

$$
\left(\frac{\partial P}{\partial T}\right)_{V}=K
$$

and $\left(\frac{\partial^{2} P}{\partial T^{2}}\right)_{V}=0$

The $a$ and $b$ parameters of the Eq. (2) are temperature dependent and the conditions showed in Eqs. (21) and (22) are not satisfied. Consequently, Eq. (2) is unable neither to describe heat capacities near the critical point nor to reproduce isochoric inflection points; this information is based on [23].

\section{Results and Discussions}

The methods and procedures for determination of $a(T)$ and $b(T)$ as well as $A$ 's and $B$ 's adjustable parameters are described above. Applying these procedures to the Eqs. (2) to (10), it is possible to calculate the parameters $A$ ' $S$ and $B$ ' $S$ of each pure substance, which are showed in Table 1, wherein the critical pressures and temperatures as well as acentric factors and deviations obtained using both the Eq. (2) and adjustable parameters $A$ 's and $B$ 's of each substance are represented. It is important to emphasize that the $T_{r}$ range adopted in present work is $0.40 \leq T_{r} \leq 1.00$ as well as the modification proposed in the EOS satisfies only its application to behavior study of situations below to or up to the critical conditions of pure compounds.

It is important to salient that the pure compounds presented in Table 1 are the same studied by [5]; inclusively, the $\operatorname{Tr}$ range employed is slightly wider, since in this (20)tk $0.40 \leq T r \leq 1.00$ (versus $0.50 \leq \operatorname{Tr} \leq 0.99$ presented in [5]); this criterion is adopted so that comparisons can be established. Using only acentric factor and critical properties of pure compounds, it is possible to calculate the pressure vapor at any temperature.

The expression used to calculate the average absolute deviations (AAD) using experimental vapor pressure of pure compounds $\left(P_{e x}\right)$ and the Eq. (2), with the application of the generalized alpha and beta functions represented by Eqs. $(7)$ and $(8)\left(P_{\text {calc }}\right)$, is represented by:

$$
A A D=\frac{\left|P_{e x}-P_{c a l c}\right|}{P_{e x}} \cdot \frac{100}{N p}
$$

Table 1. Adjustable Parameters Eqs. (7) and (8) for Eq. (2) from Pure Compounds Data.

\begin{tabular}{lcccccccccc}
\hline & $\boldsymbol{P} \boldsymbol{c}(\mathbf{b a r})$ & $\boldsymbol{T} \boldsymbol{c}(\mathbf{K})$ & $\boldsymbol{\omega}$ & $\boldsymbol{A}_{\boldsymbol{I}}$ & $\boldsymbol{A}_{\mathbf{2}}$ & $\boldsymbol{A}_{\mathbf{3}}$ & $\boldsymbol{B}_{\boldsymbol{1}}$ & $\boldsymbol{B}_{\mathbf{2}}$ & $\boldsymbol{B}_{\mathbf{3}}$ & $\begin{array}{c}\text { AAD } \\
\boldsymbol{\%}\end{array}$ \\
\hline Methane & 46.00 & 190.5 & 0.011 & 0.1407 & -0.7345 & 1.2291 & -0.1648 & 0.2489 & 0.1223 & 1.00 \\
Ethane & 48.64 & 305.4 & 0.099 & 0.0411 & -0.0730 & 0.5088 & -0.2606 & 0.7059 & -0.3949 & 0.19 \\
Propane & 42.56 & 369.8 & 0.153 & 0.0994 & -0.3777 & 1.0618 & -0.2834 & 0.8997 & -0.6646 & 0.11 \\
$n$-Butane & 37.90 & 425.2 & 0.199 & 0.0203 & 0.2426 & 0.1670 & -0.2852 & 1.0028 & -0.8531 & 0.27 \\
$i$-Butane & 36.58 & 408.1 & 0.183 & 0.0831 & -0.2041 & 0.8386 & -0.2818 & 0.9991 & -0.8655 & 0.16 \\
$n$-Heptane & 27.36 & 540.1 & 0.349 & 0.0140 & 0.5691 & -0.1722 & -0.2509 & 1.0713 & -1.1399 & 0.40 \\
$n$-Octane & 24.82 & 568.8 & 0.398 & 0.1179 & -0.0886 & 0.9658 & -0.2273 & 1.0065 & -1.1125 & 0.22 \\
Ethylene & 50.46 & 282.6 & 0.089 & 0.1145 & -0.5546 & 1.1871 & -0.2291 & 0.4847 & -0.0798 & 0.08 \\
Propylene & 46.00 & 364.9 & 0.144 & -0.0228 & 0.3851 & -0.0623 & -0.2987 & 0.9666 & -0.7538 & 0.31 \\
Argon & 48.73 & 150.8 & 0.001 & 0.1729 & -0.9537 & 1.5295 & -0.1307 & 0.0543 & 0.3321 & 0.11 \\
Xenon & 49.17 & 289.7 & 0.008 & 0.1620 & -0.8703 & 1.4325 & -0.1526 & 0.1944 & 0.2222 & 0.08 \\
Carbon Dioxide & 73.76 & 304.2 & 0.239 & 0.2226 & -1.3955 & 3.0514 & -0.0324 & -1.3031 & 2.7177 & 0.13 \\
Sulfur Dioxide & 79.84 & 430.8 & 0.256 & -0.0316 & 0.4703 & 0.0283 & -0.2318 & 0.0551 & 0.8698 & 0.41 \\
Oxygen & 50.36 & 154.6 & 0.025 & 0.1714 & -0.9394 & 1.5628 & -0.0936 & -0.2981 & 0.9169 & 0.21 \\
Nitrogen & 33.84 & 126.2 & 0.039 & 0.1621 & -0.8865 & 1.5407 & -0.0998 & -0.2900 & 0.9612 & 0.13 \\
Dichlorotetrafluoroethane & 33.03 & 418.6 & 0.263 & 0.0084 & 0.3658 & 0.1428 & -0.3095 & 1.1712 & -1.0962 & 0.23 \\
Chlorodifluoromethane & 49.85 & 369.3 & 0.221 & 0.0247 & 0.1057 & 0.5237 & -0.3052 & 0.8126 & -0.3861 & 0.18 \\
Toluene & 41.04 & 591.7 & 0.263 & -0.0038 & 0.5117 & -0.1468 & -0.2862 & 1.1230 & -1.0957 & 0.36 \\
Benzene & 48.94 & 562.2 & 0.212 & 0.0170 & 0.2724 & 0.1591 & -0.2922 & 1.0391 & -0.8987 & 0.31 \\
\hline
\end{tabular}


Table 2. Comparison between Deviations in Experimental and Calculated Vapor Pressure Using an EOS.

\begin{tabular}{|c|c|c|c|c|c|}
\hline & $\begin{array}{l}\text { Eqs. (2) and (7) }+ \\
\text { (8) }\end{array}$ & $\begin{array}{c}\text { Nasrifar and } \\
\text { Moshfeghian [5] EOS } \\
+ \text { Eqs. }(7)+(8)\end{array}$ & $\begin{array}{c}\text { Nasrifar and } \\
\text { Moshfeghian [5] EOS }+ \\
\text { (Mathias \& Copeman, } \\
{[12] \alpha \text { and } \beta \text { functions }}\end{array}$ & $\begin{array}{c}\text { Eq. (2) }+ \text { Mathias and } \\
\text { Copeman }[12] \alpha \text { and } \\
\beta \text { functions }\end{array}$ & $\begin{array}{c}\text { Nasrifar and } \\
\text { Moshfeghian } \\
\text { [5] EOS }\end{array}$ \\
\hline Compound & AAD (\%) & AAD (\%) & AAD (\%) & AAD (\%) & AAD (\%) \\
\hline Methane & 0.09 & 0.20 & 0.11 & 0.49 & 1.36 \\
\hline Ethane & 0.12 & 0.12 & 0.21 & 0.49 & 1.22 \\
\hline Propane & 0.24 & 0.64 & 0.27 & 0.68 & 0.78 \\
\hline$n$-Butane & 0.24 & 0.25 & 0.33 & 0.68 & 1.54 \\
\hline$i$-Butane & 0.26 & 0.45 & 0.30 & 0.71 & 1.20 \\
\hline$n$-Heptane & 0.21 & 0.22 & 0.27 & 0.71 & 1.77 \\
\hline$n$-Octane & 0.30 & 0.55 & 0.26 & 0.76 & 3.00 \\
\hline Ethylene & 0.14 & 0.39 & 0.20 & 0.48 & 2.00 \\
\hline Propylene & 0.19 & 0.28 & 0.26 & 0.59 & 0.53 \\
\hline Argon & 0.11 & 0.16 & 0.13 & 0.72 & 0.62 \\
\hline Xenon & 0.08 & 0.18 & 0.08 & 0.66 & 0.69 \\
\hline Carbon Dioxide & 0.18 & 0.23 & 0.57 & 1.00 & 0.59 \\
\hline Sulfur Dioxide & 0.27 & 0.28 & 0.35 & 0.72 & 1.75 \\
\hline Oxygen & 0.18 & 0.19 & 0.17 & 0.57 & 1.09 \\
\hline Nitrogen & 0.12 & 0.16 & 0.12 & 0.56 & 0.86 \\
\hline Dichlorotetrafluoroethane & 0.21 & 0.29 & 0.33 & 0.54 & 0.38 \\
\hline Chlorodifluoromethane & 0.20 & 0.22 & 0.33 & 0.54 & 1.33 \\
\hline Toluene & 0.24 & 0.25 & 0.32 & 0.69 & 1.41 \\
\hline Benzene & 0.24 & 0.26 & 0.33 & 0.72 & 0.42 \\
\hline AVERAGE & 0.19 & 0.28 & 0.28 & 0.65 & 1.19 \\
\hline
\end{tabular}

in which $N p$ is the number of experimental vapor pressure points of each pure compound. In present work the minimum $N p$ value employed is 20 . The AAD values are showed in Table 2 .

In Table 2, besides to deviations calculated using the proposed modified equation of state (column 2), it is possible to observe deviations obtained between calculated and experimental vapor pressure data, using the following combinations: Nasrifar and Moshfeghian EOS [5] + Eqs. (7) and (8) (column 3), Nasrifar and Moshfeghian EOS [5] + Mathias and Copeman alpha and beta functions [12] (column 4), Eq. (2) + Mathias and Copeman $\alpha$ and $\beta$ functions [12] (column 5), and Nasrifar and Moshfeghian EOS [5] (column 6).

Comparing each pure compound, it is possible to observe that the calculated deviations presented in column 2 are smallest than those presented in the columns 3 to 6 . In relation to the average values deviation (presented in last line of the Table 2), the value obtained in the column 2 is also smaller than those showed in another columns. These features can be attributed to the proposed modifications presented in Eq. (2): a repulsive term with better physical consistency and the attraction and repulsion parameters accounted temperature dependent.

Another likely cause of the deviations improvement values in the calculations of the vapor pressure data can be due to substitution of the alpha function accounted in original Nasrifar and Moshfeghian [5] by the $\alpha(T r)$ and $\beta(T r)$ functions showed in the Eqs. (7) and (8). This situation can help to verify if the use of an alpha and beta functions more elaborated mathematically, such as the Mathias and Copeman expression [12], can enhance the representation of the pure compound vapor pressure data. In relation to the average deviations presented in last line of the Table 1, its values are similar in both columns 3 and 4 . This reveals that if ever alpha and beta functions here studied were employed, the improvement label of the results is satisfactory.

Comparatively to the column 6 , the deviations obtained in the column 5 are better. This shows that to employ a more consistent repulsive term, and alpha and beta functions, can help to improve the vapor pressure calculated data. However, the study of these modifications show that the Eq. (2), combined to Mathias and Copeman [12] mathematical structure, not improved the results in relation to those presented in the columns 2 to 4 . Perhaps the Mathias \& Copeman alpha and beta functions are inadequate in combination with the Carnahan and Starling [15] repulsive term, using the attractive term proposed by Nasrifar and Moshfeghian [5]. This alternative can be treated as a real possibility of verification, since the combination of the Eqs. (2), (7) and (8) brought satisfactory results in the deviation calculations of vapor pressure. In this way, the influence of the attractive term must be studied carefully.

In the reference [5], as well as in works of Coquelet et al. [14] and Chiavone-Filho et al. [24], EOS and/or generalized alpha functions are proposed. In these works, the $\mathrm{AAD}$ in terms of vapor pressure for various pure compounds are showed and compared to the deviations obtained by Soave [2] and Peng and Robinson [3] EOSs. (To illustrate, methane is accounted as an example and percentage deviations values are showed in [2-3], respectively: in the Nasrifar and Moshfeghian [5], $1.71 \%$ and $2.08 \%$; in Coquelet et al. [14], $1.54 \%$ and $0.70 \%$; in Chiavone-Filho et al. [24] - only for Peng and Robinson [3] $-2.32 \%$.)

Taking to consideration the deviations values obtained using the Eqs. (2), (7) and (8), it is possible to observe a sensible improvement in the results when compared with deviations presented in [2-3], [5], [14], and [24]. This improvement can be attributed to the substitution of original repulsive term in equation (1) by Carnahan-Starling repulsion term, whose theoretical importance and correct representation of intermolecular repulsions behavior in real fluids is confirmed in several works in the literature [6], [13], and [15-18].

Another fact contributes to this improvement: the correct choice of the EOS and generalized functions temperature dependence as well as of the $T r$ range studied. It is well known that generalized alpha and beta functions not always represent accurately vapor pressure data behavior for $\operatorname{Tr}$ near 1.0. In present work, the generalized functions, employed in the Eq. (2), can be considered as an 
adequate choice due to its capability in to reproduce vapor pressure values in critical conditions, i.e., $\operatorname{Tr}=1.0$, demonstrated in the deviations showed in the first column of the Table 2 .

It is noteworthy that the reduced temperature is limited to 1.0 in present work because our intention is: a) to verify the applicability of a modified version of the Mathias and Copeman alpha function [12], in which accounts square roots in its structure, preventing negative numbers be employed in the calculations (for this reason $\operatorname{Tr}$ should not exceed 1.0), and (b) to apply the results only for studies involving conditions well below to the critical conditions (such as liquid phase and lower pressures, around 1.0 bar).

In Eqs. (24) to (29) are represented the parameters to calculate the generalized alpha and beta functions, employing Eqs. (9) and (10), as a function of the acentric factor.

$$
\begin{aligned}
& A_{1}=0.17649-1.15920 \omega+2.34959 \omega^{2} \\
& A_{2}=-0.96461+7.26811 \omega-11.31294 \omega^{2} \\
& A_{3}=1.53234-7.38994 \omega+12.59803 \omega^{2} \\
& B_{1}=-0.12484-1.19070 \omega+2.47238 \omega^{2} \\
& B_{2}=0.03375+4.35137 \omega-5.71397 \omega^{2} \\
& B_{3}=0.38277-3.48972 \omega+0.68399 \omega^{2}
\end{aligned}
$$

Interesting predicted result obtained, using Eq. (2), for the critical compressibility factor: $Z c=0.2809$; it is in good agreement with the experimental values reported for the majority of gases [27]. This value is physically more adequate than those obtained in [12] for the modification of van der Waals and Soave EOSs repulsive terms. In [17], after the changing of the repulsive term in the Dieterici EOS proposed in [15], it was found a value for $Z c=0.2705$, which is very close to obtained here.

The parameters specific to each pure compound and the generalized parameters can be used to calculate the vapor pressure of the pure compounds studied in present work. Considering a pure compound in the Tables 1 and 2 it is possible to note, in some cases, that the calculated deviations are higher when the parameters used are specific to it; in another cases, to employ generalized parameters to the calculations generate deviations greater than those obtained when specific parameters are accounted. In this way, it is possible to choice what parameters must be used, according to the convenience of the calculations and of the results to be obtained. In some situations, the requirement is to have precision in the calculated results, and in another cases, it is desirable to facilitate the calculations, even if occurs the accuracy decrease of the results. Thus, it is up to the researcher to choose the method to be adopted in the calculations of the thermophysical properties.

\section{Conclusions}

Substitution of the repulsion term in a cubic equation of state by an appropriate theoretical repulsive term using generalized alpha and beta functions proposed by Mathias and Copeman [12] and Eqs. (7) and (8), shows a possibility to improve the correlative capability of an EOS in vaporpressure data calculations for non-polar pure compounds, especially for behaviors up to critical conditions $(0.40 \leq \mathrm{Tr}$ $\leq 1.00)$; these calculated data are in satisfactory agreement with the experimental data of non-polar pure compounds vapor pressure studied in this work.

The deviations observed shows that the modified EOS proposed here is adequate and can be compared to the correlative capability of the equations of state proposed by Soave [2] and Peng and Robinson [3], besides to the results obtained by EOSs presented in [12], [6], [25-26], [14]. The adequate choice of an equation of state as well as generalized alpha function, associated to account the covolume temperature dependent, contributes to decrease the differences between experimental and calculated data of thermophysical properties of equilibrium.

According to the Deiters and De Reuck guidelines [23], some criteria must be accounted for the development of equations of state to describe the PVT pure compounds data behavior. As reported above, at least three criterions presented in this publication were satisfied by the EOS proposed here. However, we know that it is necessary a detailed study in relation to another criterion to reinforce the validation of the applicability range of an equation of state. Inclusively, the next step of our research is related to the calculations of the liquid and vapor volume, since previous studies realized shows satisfactory agreement with experimental data for this pure compound property, by using the EOS here reported.

\section{Acknowledgement}

Ricardo F. Checoni wishes to thank CNPq (Conselho Nacional de Desenvolvimento Científico e Tecnológico) for the financial support received in the form of a pos-doctoral fellowship. M. Aznar is the recipient of a CNPq fellowship.

\section{References}

[1] M. M. Abbot, Thirteen Ways of Looking at the van der Waals Equation. Chem. Eng. Progress, 2, 25-37, 1989.

[2] G. Soave, Equilibrium constants from a modified Redlich-Kwong equation of state. Chem. Eng. Sci., 27, 1197-1203, 1972.

[3] D-Y. Peng, D. B. Robinson, A New Two-Constant Equation of State. Ind. Eng. Chem. Fundam., 15, 59-64, 1976.

[4] Y. S. Wei, R. J. Sadus, Equations of State for the Calculation of Fluid Phase Equilibria. AIChE J, 46, 169196, 2000.

[5] K. Nasrifar, M. Moshfeghian, A new cubic equation of state for simple fluids: pure and mixture. Fluid Phase Equilibria, 190, 73-88 (2001).

[6] C. H. Twu, J. E. Coon, J. R. Cunninghan, A new generalized alpha function for a cubic equation of state Part 1. Peng-Robinson equation. Fluid Phase Equilibria, 105, 49-59, 1995.

[7] M-R. Riazi, G. A. Mansoori, Simple equation of state accurately predicts hydrocarbon densities. Oil and Gas Journal, 12, 108-111, 1993.

[8] S. Hajipour, M. Edalat, A new hard sphere cubic equation of state for predicting fluids' properties and vapor-liquid phase equilibrium calculations. Journal of Phase Equilibria and Difusion, 29, 322-332, 2008.

[9] R. F. Checoni, S. P. Ravagnani, (2013). Studies about Equation of State for Pure Associated Fluids: Temperature Dependent Co-Volume Accounting a 
Physically Consistent Repulsive Term, Int. J. Thermo., 16, 20-27, 2013.

[10] H. Toghiani, D. S. Viswanath, A Cubic Equation of State for Polar and Apolar Fluids. Ind. Eng. Chem.. Process Des. Dev., 25, 531-536, 1986.

[11] Z. Xu, S. I. Sandler, Temperature dependent parameters and Peng-Robinson equation of state. Ind. Eng. Chem. Res., 26, 601-606, 1987.

[12] P. M. Mathias, T. W. Copeman, Extension of the Peng-Robinson Equations of State to Complex Mixtures: Evaluation of the Various Forms of the Local Composition Concept. Fluid Phase Equilibria, 13, 91108 (1983).

[13] M. A. Trebble, P. R. Bishnoi, Development of a new four-parameter cubic equation of state. Fluid Phase Equilibria, 35, 1-18, 1987.

[14] C. Coquelet, A. Chapoy, D. Richon, Development of a New Alpha Function for the Peng-Robinson Equation of State: Comparative Study of Alpha Function Models for Pure Gases (Natural Gas Components) and Water-Gas Systems. Int. J. Thermophysics, 25, 133-158, 2004.

[15] N. F. Carnahan, K. E. Starling. Intermolecular Repulsions and the Equation of State for Fluids. AIChE $J, 18,1184-1188,1972$.

[16] R. De Santis, F. Gironi, L. Marrelli, Vapor-Liquid Equilibrium from a Hard-Sphere Equation of State. Ind. Eng. Chem. Res. 15, 183-189, 1976.

[17] R. J. Sadus, Equations of state for fluids: The Dieterici approach revisited. J. Chem. Phys., 115, 1460-1462, 2001.

[18] R. J. Sadus, New Dieterici-type equations of state for fluid phase equilibria. Fluid Phase Equilibria, 212, 3139, 2003.

[19] R.H. Perry, D. W. Green, Perry's Chemical Engineers' Handbook, $7^{\text {th }}$ Edition, McGraw Hill, USA, 1997.
[20] N. B. Vargaftik, Handbook of Physical Properties of Liquid and Gases (Pure Substances and Mixtures), $2^{\text {nd }}$ Edition, John Willey, D.C, 1975.

[21] C. F. Beaton, D. Ambrose, E. Brunner, M. W. Chase, J. R. Downey, G. Hobson, A. E. Humphreys, D. T. Jamieson, S. R. Knight, M. Schoenberg, H. J. White, Jr. Ortobaric densities and molar volumes of liquids, Engineering Sciences Data Unit - ESDU. Eng. Sci. Data Item Nr. 87010, 1987.

[22] C. F. Beaton, D. Ambrose, H. J. Foxcroft, G. Hobson, D. T. Jamieson, S. R. Knight, G. M. Rowell, M. Schoenberg, H. J. White, Jr. Vapor pressures and critical points of liquids, Engineering Sciences Data Unit - ESDU. Eng. Sci. Data Item Nr. 84022 and 84028 Vapor Pressure Data, 1984.

[23] U. K. Deiters, K. M. De Reuck, Guidelines for publications of equations of state - I. Pure Compounds. Pure \& Applied Chemistry, 69 1237-1249, 1997.

[24] O. Chiavone-Filho, P. G. Amaral-Filho, D. N. Silva, L. R., Terron, Alpha Function for a Series of Hydrocarbons to Peng-Robinson and van der Waals Equations of State. Ind. Eng. Chem. Res., 40, 62406244, 2001.

[25] Y. S. Wei, R. J. Sadus, E. U. Franck, Binary mixtures of water + five noble gases: comparison of bimodal and critical curves at high pressures. Fluid Phase Equilibria, 123, 1-15, 1996.

[26] C. J. Kedge, M. A. Trebble, Improvements to a new equation of state for pure components. Fluid Phase Equilibria, 215, 91-96, 2004.

[27] L. V. Yelash, T. Kraska, Investigation of a generalized attraction term of an equation of state and its influence on the phase behavior. Fluid Phase Equilibria, 162, 115-130, 1999. 